\title{
Perceptions of the Value of Clinical Pharmacy Medication Review for Women During Early Pregnancy
}

\author{
Minna Lee, PharmD; Deanna Kurz, BA; Sarah Schwiesow, PharmD; Thomas Delate, PhD, MS; \\ Stephanie Campbell, PharmD; Kara Rivera, PharmD; and Kari Olson, PharmD
}

\begin{abstract}
BACKGROUND: The benefit of continuing medications to prevent or treat illness is often overlooked, since pregnant women tend to overestimate the teratogenic risk of medications. Pharmacists can serve as a resource to prescribers and pregnant women with their knowledge of the appropriate use and management of medications during pregnancy. Little information exists on the value women place on pharmacists' medication management during pregnancy.
\end{abstract}

OBJECTIVE: To assess pregnant women's perceptions of an ambulatory care clinical pharmacist (CP) medication review service during early pregnancy that provided education regarding the risks and benefits of medication use during pregnancy.

METHODS: This was a qualitative study of pregnant women using semistructured telephone interviews performed between December 12, 2018, and January 18, 2019, and conducted in an integrated health care delivery system. Potential participants were identified from CP encounter records. Consented English-speaking women aged $\geq 18$ years participated in an up to 30-minute interview within 1 week of the CP encounter. Interviews were professionally transcribed and coded line by line using the constant comparison method with grounded theory used to gain insight into participants' perspectives.

RESULTS: 62 women were invited to participate in semistructured telephone interviews of whom $24(39 \%)$ completed the interview. Three main themes emerged from the qualitative analysis: satisfaction with the service, comfort with medication use during pregnancy, and connectedness to the health care team. Overall, the CP medication review and education service was perceived positively by the participants. Participants reported satisfaction in the quality, timeliness, and convenience of the service and found it beneficial to have their medications reviewed early during pregnancy to assist in medication use decisions before their first obstetric visit.

CONCLUSIONS: CP medication review provided a comforting, valuable service for women during early pregnancy when medication-taking decisions can feel exigent.

J Manag Care Spec Pharm. 2020;26(10):1301-08

Copyright $\odot 2020$, Academy of Managed Care Pharmacy. All rights reserved.

\section{What is already known about this subject}

The benefit of continuing medications to prevent or treat illness can be overlooked, since pregnant women often overestimate the teratogenic risk of medications.
Pharmacists can serve as a resource for prescribers and pregnant women because of their knowledge of the appropriate use and management of medications during pregnancy, but little is known of the value that women place on pharmacists' medication management during pregnancy.

\section{What this study adds}

This qualitative study of pregnant women's perceptions of an ambulatory care clinical pharmacist medication review service during early pregnancy identified 3 main themes: satisfaction with the service, comfort with medication use during pregnancy, and connectedness to the health care team.

Overall, the clinical pharmacist service was perceived positively by the participants.

Participants found it beneficial to have their medications reviewed early during pregnancy to assist in medication use decisions.

T he prevalence of medication use during pregnancy is increasing. Over the past 30 years, the proportion of women receiving a prescription medication during pregnancy has doubled. It is estimated that $90 \%$ of women use at least 1 medication at some point during pregnancy. ${ }^{1-3} \mathrm{~A}$ cohort study in the United States reported that $35 \%$ of nulliparous women were taking $\geq 5$ medications in addition to over-thecounter (OTC) medications during pregnancy. ${ }^{4}$ Increased awareness and treatment of preexisting chronic diseases and conditions that may develop during pregnancy (e.g., asthma, depression, cardiovascular conditions, and diabetes) have likely contributed to the increased medication use. ${ }^{5}$

Pregnant women are excluded routinely from clinical trials, so information about the safety of medication use during pregnancy is limited. ${ }^{6}$ While many medications do not disturb the development of the embryo or fetus, there are misconceptions that all medications should be stopped once pregnancy is confirmed. ${ }^{7}$ A survey of obstetrician-gynecologists reported that $90 \%$ and $63 \%$ received questions from their pregnant patients about the effects of prescription and OTC medications on the fetus, respectively. ${ }^{8}$ The benefit of continuing medications to prevent or treat illness is often overlooked, since women can overestimate the teratogenic risk of medications., ${ }^{5,9-11}$ Discontinuation of necessary and often safe medications can 
lead to suboptimal control of preexisting or acute conditions and can jeopardize the health and safety of mother and fetus. $5,7,9,11$

As the medication therapy experts, clinical pharmacists (CP) are essential members of a patient's health care team. ${ }^{12}$ CPs can serve as a resource to prescribers and pregnant women because of their knowledge of the appropriate use and management of medications during pregnancy. ${ }^{11}$ The limited studies of $C P$ interventions in medication use in pregnancy are primarily focused on $\mathrm{CP}$ potential and actual roles and not on women's perceptions of $\mathrm{CP}$ medication management during pregnancy. ${ }^{5-7,13}$ Little information exists regarding the value that women place on medication management by CPs during pregnancy. The objective of this study was to assess pregnant women's perceptions of a CP medication review and education service for newly pregnant women. Information obtained from this study can provide insights into the importance that pregnant women place on $\mathrm{CP}$ outreach and knowledge of the perceived usefulness of such a service for use by policymakers.

\section{Methods}

\section{Study Design and Setting}

This was a qualitative study of pregnant women using semistructured telephone interviews performed between December 12, 2018, and January 18, 2019. The study was conducted at Kaiser Permanente Colorado (KPCO), an integrated health care delivery system that provided care to more than 640,000 members in 30 medical offices at the time of this study.

A CP medication review service for pregnant women was developed at KPCO in early 2017. Before that time, medication use was not consistently assessed for potential quality and safety issues during pregnancy. For the service, obstetric nurses identified and triaged pregnant women who were receiving at least 1 prescription medication. The nurse contacted the women before their first obstetric visit, typically between the eighth and twelfth week of pregnancy (i.e., newly pregnant), to review their medication history, including OTC medications. The information collected was forwarded via the electronic health record (EHR) for a comprehensive medication review by an ambulatory care $\mathrm{CP}$.

The $\mathrm{CP}$ reviewed available resources on the specific medications reported by the pregnant women. The $\mathrm{CP}$ then contacted the women by telephone and/or email communication to discuss and educate about the risks and benefits of medication in early pregnancy and address any concerns or questions about medication use during pregnancy. The encounter was documented in the EHR, sent to the appropriate practitioner, and made available for the health care team at the first obstetric visit. The CP was available for further medication-related consultation with the obstetric care team. Potential research

\section{TABLE 1 Interview Guide}

\section{All Participants}

1. Describe your interaction with the clinical pharmacist (CP) regarding your prescription and OTC medications in pregnancy.

2. How did [CP name] make you feel during your conversation about your medications?

3. How did you feel about the information that [CP name] provided during this conversation?

4. How did your interaction with [CP name] influence your decision for medication use during pregnancy?

a. Based on the pharmacist's recommendations, were there any medications you:

i. Stopped?

ii. Started?

iii. Restarted?

iv. Possibly were going to stop but decided to continue?

5. Describe any benefits or drawbacks to having the pharmacist talk to you early in your pregnancy.

6. What additional questions or concerns do you wish the pharmacist would have addressed during the call?

7. How do you think the call from the pharmacist might influence your first OB visit?

Subsequent Pregnancy Only

8. How did you feel about your interaction with the pharmacist in comparison to previous pregnancies where you did not have this service?

$C P=$ clinical pharmacist; $O B=$ obstetric; $O T C=$ over the counter.

participants were identified from the $\mathrm{CP}$ encounters that were documented in the EHR. All aspects of this study were reviewed and approved by the KPCO Institutional Review Board before data collection.

\section{Participants}

Newly pregnant women aged $\geq 18$ years and who had a CP medication review documented in the EHR were eligible for inclusion. Non-English speakers were excluded because the investigators were not multilingual. No participant compensation was provided.

\section{Study Procedures}

Potential research participants were identified from the CP clinical encounter records. The index date was the day of the medication review by the CP. There were up to 3 contact attempts on 3 separate days to schedule a telephone interview. Interviews were completed within 3 weeks from the index date. The interviews were conducted by 2 study investigators (Lee and Kurz) not involved in the service. Informed consent to participate and be audio-recorded was obtained verbally before interview administration.

The interviews lasted up to 30 minutes. All interviews were professionally transcribed and stored in a secure database. Interviews from a convenience sample of up to 24 women were conducted to obtain response saturation. ${ }^{14}$ 


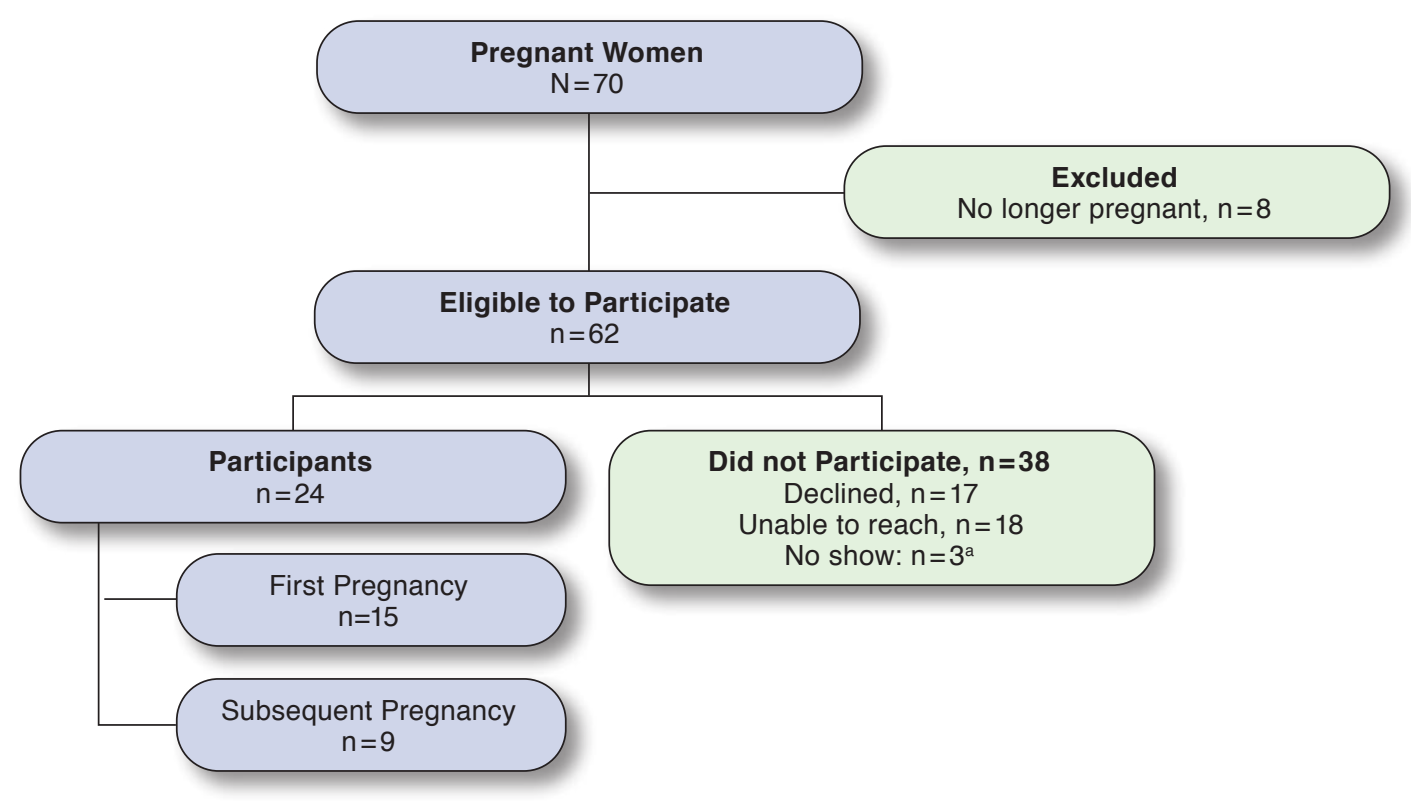

a Agreed to participate but did not follow up on scheduled telephone interview.

\section{Data Collection}

An interview guide (Table 1) was designed based on the clinical experiences of the study team and the literature. ${ }^{6,14-17}$ The questions were designed to elicit participants' perceptions of the value of their interactions with the $\mathrm{CP}$ and the information provided including their insights into the service's benefits, shortcomings, and challenges. The semistructured interview allowed for probing questions when interviewers required additional clarification or engaged in a deeper discussion of the participants' perspectives. Participants were interviewed until it was evident that new concepts or themes were unlikely to emerge from further interviews. ${ }^{14}$ Characteristics of potential participants (e.g., date of birth, race, and health plan), comorbidities, and medication-dispensing information were obtained from KPCO's administrative and claims databases.

\section{Study Outcomes}

The primary outcome was pregnant women's perceptions of the value of the CP medication review service and any subsequent medication education provided. The secondary outcome was a description of the prescription and OTC medications that the participants reported taking.

\section{Data Analysis}

Age was determined as of the index date. Characteristics, comorbidity, and medication-dispensing information was obtained during the 6 months before the index date. Characteristics per participant regarding number of medications reviewed and medication by disease state were determined as of the index date. A chronic disease score, a validated measure of the burden of chronic illness, was calculated for each woman using ambulatory prescription medication dispensings. ${ }^{18}$ The presence of specific comorbidities was determined using the Quan adaptation of the Charlson Comorbidity Index. ${ }^{19}$

Information was reported as means and standard deviations for interval-level and percentages for nominal and ordinal data. Characteristics were compared between participants and nonparticipants with chi-square tests of association and Fischer's exact tests and 2-sample t-tests for nominal- and ordinal-level and interval-level data, respectively.

Qualitative analysis was facilitated using ATLAS.ti 8.0 software (ATLAS.ti Scientific Software Development, Berlin, Germany). Data collected were reviewed independently by 2 investigators (Lee and Kurz). Grounded theory was used to gain insight into participants' perspectives. ${ }^{20}$ Interview responses were coded (i.e., assessed to identify patterns and relationships) line by line using the constant comparison method: Codes were refined, and new codes emerged when text segments did not align with previous code definitions. ${ }^{20,21}$ Deidentified data were then shared with other investigators to allow for an independent review of the codes and relationships. Study investigators peer-reviewed codes and conducted iterative readings of the transcripts to redefine codes. 


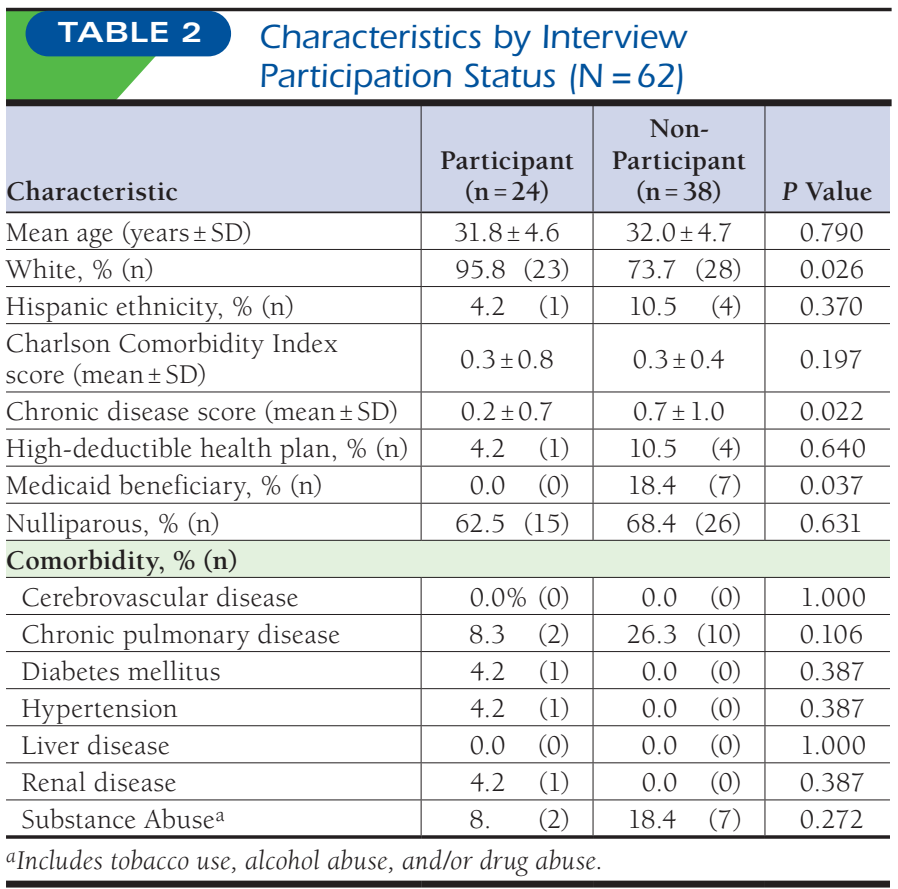

Similar codes were grouped into categories, and similar categories were linked to determine emerging themes along with supporting quotes. Emphasis was made towards identification of themes related to meanings, concept definitions, characteristics, and descriptions of the participants' perceptions. Codes, categories, emerging themes, and gaps or inconsistencies in the codes assigned to text were reviewed. This served as an overall quality check during data analysis.

\section{Results}

Seventy pregnant women were identified during the study period: 62 (88.6\%) were eligible for outreach, and 24 (38.7\%) consented and completed interviews (Figure 1). This was the first pregnancy for 15 participants (62.5\%). Compared with nonparticipants, participants were more likely to be White, not a Medicaid beneficiary, and have a lower burden of chronic disease (all $P<0.05$; Table 2). Participants reported taking a mean of 4 medications (2 prescription and 2 OTC medications or supplements; Table 3). Participants were receiving prescription medications predominately for anxiety or depression, allergic rhinitis, and asthma and for various OTC medications or supplements for no specific disease state. The mean (SD) time from the CP encounter to first obstetric visit was 11 (3) days. Three main themes emerged in the qualitative analysis: satisfaction with service, comfort with medication use during pregnancy, and connectedness to the health care team.

\section{Satisfaction with Service}

Multiple participants expressed that their encounter with the $\mathrm{CP}$ was very positive. They stated how the $\mathrm{CP}$ made them feel very comfortable, cared for, confident, and secure. "She definitely made me feel more comfortable and... you could definitely tell through the conversation that she cared and I really liked that, too, that she cared about my success and doing well" (Participant \#26).

Multiple participants reported that speaking to the $\mathrm{CP}$ made them feel at ease, as it reduced the stress of whether their medications were harmful to their baby. "I was extremely thankful and it really-I've talked with quite a few people about it already. It just made me feel so much better and at ease with stuff versus the other conversations that really kind of stressed me out" (Participant \#42).

Similarly, a few participants expressed how appreciative they were since the conversation was nonjudgmental and they could be frank about all the questions they had. "I felt very listened to. I felt like-I didn't feel rushed. I felt like there was plenty of time to ask all of my questions. I felt like it was very nonjudgmental. It ... wasn't like, 'Oh, you can't take this, you're pregnant.' It was like, 'What's the problem you're trying to solve?' and 'We'll find you a medication that's going to work for you'” (Participant \#14).

Numerous participants reported that the CP was very knowledgeable regarding medication use in pregnancy, allowing them to feel confident in the CP's expertise, as well as making them feel valued and supported in their care. "[CP name] was very knowledgeable and informative on everything she told me about the medication that I'm on currently for depression. And she explained things very well to me. And I could tell she was very knowledgeable with what she was talking about" (Participant \#22).

Most participants were appreciative of the medication review, since it included not only prescription medications but also OTC medications and supplements. "I really appreciated her thoroughness and taking-even though it was just a supplement - but taking it seriously and giving me really good information" (Participant \#49).

Participants expressed how pleasantly surprised they were to receive a call and be provided useful information without waiting until their obstetric appointment. "I loved that I was able to just have that conversation with her over the phone and I didn't have to wait until an appointment and that I was taken care of right away" (Participant \#43).

\section{Comfort with Medication Use During Pregnancy}

Most participants conveyed how speaking to the CP influenced their decisions on medication use throughout their pregnancies. "I had actually been wondering if I needed to stop some of my asthma medications and I'd been trying to wean myself off. She [CP] reassured me that most asthma medications are pretty safe and that it would be more unsafe to go off of them" (Participant \#34).

A common response from participants was the reassurance and confidence they felt in their decisions to continue or stop a medication after the medication review. While some 


\begin{tabular}{|c|c|}
\hline Characteristic & Participant $(n=24)$ \\
\hline Total medications (mean \pm SD) & $3.8 \pm 2.6$ \\
\hline Prescriptions (mean \pm SD) & $1.8 \pm 1.7$ \\
\hline OTC medications/supplements (mean \pm SD) & $2.0 \pm 2.1$ \\
\hline Medication by disease state, $\%$ (n) & Total medications $(\mathrm{n}=91)$ \\
\hline Acne & $4.4 \quad(4)$ \\
\hline Allergic rhinitis & $8.8 \quad(8)$ \\
\hline Anxiety/depression & $13.2(12)$ \\
\hline Asthma & $7.7 \quad(7)$ \\
\hline Atopic dermatitis & $1.1 \quad(1)$ \\
\hline Constipation & $5.5 \quad(5)$ \\
\hline Diabetes & $3.3 \quad(3)$ \\
\hline Gastroesophageal reflux disease & $3.3 \quad(3)$ \\
\hline Genital herpes & $2.2 \quad(2)$ \\
\hline Hypertension & $4.4 \quad(4)$ \\
\hline Hypothyroidism & $1.1 \quad(1)$ \\
\hline Insomnia & $3.3 \quad(3)$ \\
\hline Migraines/headache & $5.5 \quad(5)$ \\
\hline Multiple sclerosis & $2.2 \quad(2)$ \\
\hline Nausea/vomiting & $4.4 \quad(4)$ \\
\hline Polycystic ovarian syndrome & $2.2 \quad(2)$ \\
\hline Other (OTC medications/supplements) & $27.5(25)$ \\
\hline
\end{tabular}

participants decided to stop taking a medication given lack of safety data, others decided to continue. Overall, participants reported feeling empowered and more confident about their decisions to continue, stop, or start a medication following their discussions with the CP. "I was taking a medication regularly, not like - not by obligation but by choice and then I had stopped taking as soon as I found out I was pregnant, and then basically, now, I have 2 options. I can take it when I need or take it regularly. So, she gave me the choice to make that decision on my own" (Participant \#1).

One participant reported strong appreciation and support with her medication decision from the CP. "The medication that I'm taking is really important, more towards the end of my pregnancy so that I can have a vaginal birth. . . Knowing what my options are early on really made me feel comfortable and just gave me like more freedom to feel happy and not nervous" (Participant \#1).

One participant noted that the information she received was timely and beneficial to not only reduce her medicationtaking fears but also reassure her that any potential medication risk was addressed before her first obstetric visit. "I think it's extremely beneficial because you never quite know with supplements or other things you're taking so it's really nice to have very quick feedback and not have to wait for an appointment" (Participant \#49).

Other participants expressed feeling more prepared for other situations after speaking with the $\mathrm{CP}$ (e.g., when the CP told them what medications to take for headaches or allergies or what to use/do when feeling nauseous). Most participants did not have any additional questions or concerns that were not addressed by the CP.

\section{Connectedness to the Health Care Team}

Several participants acknowledged that their conversations with the $\mathrm{CP}$ affected their perception of care that they were receiving (e.g., making them feel welcomed and connected to their health care team). "I'm a new member, I just felt really welcomed, and like I already have a care team looking out for me" (Participant \#14).

Another participant appreciated the recommendations and communications to other health care team members made by the CP. "I had really bad pre-eclampsia with my first pregnancy for the last couple of months and even about 6 months after. My blood pressure was all over the place, and it kind of left me feeling kind of wonky, so she recommended taking baby aspirin, like an $81 \mathrm{mg}$ aspirin every day. And what was great about our interaction was she actually checked with my doctor and my OB/GYN and then called me back. So, it was like 3 different people telling me that that was a safe medication to take" (Participant \#39).

Several participants expressed positive remarks regarding not only the initial CP's contact, but also a perceived benefit from the accessibility of the $\mathrm{CP}$ in addition to their prescribers for any future medication-related questions. "She gave me her direct line and so I thought that was really helpful because she said I could use her as a resource with any questions I have throughout pregnancy. So, I thought that was really comforting to know that I have someone I can reach out to if I do have a medication question and I don't necessarily have to always reach out to just my OB/GYN for that" (Participant \#46).

Participants reported better interactions with the CP than previous encounters with their health care team during a previous pregnancy. "She really helped way more than a doctor has ever helped to explain it to me and then to make me feel comfortable doing what I thought was the safest for myself" (Participant \#42).

One participant described the prejudice and discomfort she felt during her previous pregnancy and that her encounter with the CP made her feel like a normal pregnant woman. "She made me feel like my health was normal. ... I've had herpes, and in the past, I felt like doctors treated me like there was something wrong with me, and I feel like she just made me feel like 'No, ... lots of women have this and...you can still have a natural birth and everything"' (Participant \#1).

Several participants expressed satisfaction with the effect that the review could have on their obstetric visit, including a feeling of empowerment that allowed them to focus on other nonmedication-related questions or concerns. "I think that I'll go into the visit feeling in control of my situation, which is really important to me. I like to be very informed and aware of what's going on with my body" (Participant \#1). 
Many participants expressed gratitude that their obstetric practitioner would know what medications they were currently taking and what they were not taking that may be unsafe. "I think that if anything it would have a positive effect because when I go to see the $O B$, we'll kind of be on the same page as far as what medications I'm currently taking and that I'm not taking anything that could be bad for the baby" (Participant \#61).

Some participants did not appear to understand the function of an integrated health care delivery system or the purpose of the medication review before the first obstetric visit. "I hope the $\mathrm{OB}$ agrees that I can keep taking the medication. . . I'm going to run it by him" (Participant \#55).

One participant was uneasy and felt unsure whether all of her health care practitioners would be contacted about the medication use plan the $\mathrm{CP}$ described. She stated, "It would be more comfortable talking to my actual provider before going off of any medication because I feel like at the moment, I'm... being directed from multiple directions and I don't know if everybody is talking to each other" (Participant \#50).

Another participant expressed redundancy with the service. "I... feel that I'm going to ask the same exact questions at my other appointment and so just repetitive" (Participant \#40).

\section{Discussion}

This was a qualitative analysis of pregnant women's perceived value of their interaction with and information provided by a CP medication review service before their first obstetric visit. We identified 3 themes during our interviews with the participants: satisfaction with service, comfort with medication use during pregnancy, and connectedness to the health care team.

Participants' satisfaction with the $\mathrm{CP}$ medication review and education service was overwhelmingly valued based on participants' perceptions of the quality, value, and convenience of the one-on-one encounters. Participants' reassurance and confidence in their decisions to continue or stop a medication after the medication review was a value that was commonly articulated. Furthermore, participants' sense of improved coordination of care between the pharmacist and their health care teams compared with previous pregnancies were valued. Participants also valued the timing of the call, since it came before their first obstetric visits and allowed for a conversation about their medications, while increasing their feelings of support during pregnancy.

These findings are important because this is one of the first studies to assess an ambulatory CP service for maternal care, and our findings suggest that implementation of $\mathrm{CP}$ medication review and education in a health care system would be highly valued by pregnant women who take medications.

Our findings add to the limited literature on the provision of prenatal care by pharmacists. The high satisfaction we identified was similar to that reported in the SafeStart feasibility study, where the majority of women reported that a consultation with a pharmacist was most useful when tailored to their needs and would recommend the consultation to other pregnant women. ${ }^{22}$ The majority of the literature on prenatal care by pharmacists has focused on the pharmacist's role in medication management and education for pregnant women. ${ }^{5,6,9,13,23-25}$

Little has been reported on the value that pregnant women place on pharmacists or other health care practitioners who provide information on medication use before or during pregnancy. One study conducted focus groups with women of reproductive age to assess their expectations of counseling by health care practitioners on teratogenic medication use. ${ }^{26}$ Women valued discussions about the teratogenic potential of medications and information about all potential outcomes for a fetus. ${ }^{26}$ The women reported that the counseling should be private, have sufficient time to discuss the topic, and involve a trusting relationship with their health care practitioners. ${ }^{26}$

Our findings reflect similar preferences of pregnant women who valued the privacy, comprehensiveness, and the knowledge that the CP medication review service provided. Another study interviewed 47 pregnant women regarding their attitudes towards "surveillance medicine," including aspects of receiving information related to risks. ${ }^{27}$ The 9 women whose typology was defined as "striving for certainty," reported valuing medical information and expert opinion. ${ }^{27}$ While the preferences were not specific to CPs, they are congruent with our participants, who valued the $\mathrm{CP}$ medication review for the knowledge and expertise in medication use imparted during pregnancy.

Pregnancy is an inherently anxious time for women, since the risks and limits of control over malformations or diseases are exacerbated by prenatal care and screenings. ${ }^{27,28}$ The perceptions of our participants of the value of the CP medication review may be related to the participants' reported increased confidence in their decisions to continue medication use because the CPs were knowledgeable in prescription medications, OTC medications, and supplements and were clear about the lack of safety data, when applicable, with medications. These CPs likely empowered participants to make better informed decisions, even if the data were scarce on the relationship between medication and pregnancy outcomes.

By providing women with the education and information regarding the risks and benefits of continuing or stopping each prescription, OTC medication, or supplement, most participants expressed that they were at ease, informed, and confident to be able to make an educated decision regarding their care and how it would affect their safety and that of the fetus. Our findings were similar to other studies where counseling reduced concerns and misconceptions about medications in pregnancy. ${ }^{9}$

The way information was communicated to the participants was also important, since positively framed information has been associated with a lower perception of risk. ${ }^{9,29}$ The 
transparency of the information that the CPs provided, whether or not data were plentiful, not only reduced the uncertainty many participants had regarding medications in pregnancy but allowed them to make an informed, shared decision. In addition, leaving open a line of direct communication and reinforcing that information will be communicated with other health care team members appeared to influence participants' perceptions of the service.

The finding that some participants did not understand the integrated health care delivery system model, in which the CP and prescribers are in communication within the same EHR, provides an area of opportunity for additional education. The $\mathrm{CP}$ could include a brief explanation of how information will flow to the health care team during the medication review outreach, since the EHR communication system could help eliminate hesitance by the $\mathrm{CP}$, prescriber, and participants and barriers to ensuring medication safety during pregnancy. ${ }^{6}$ Furthermore, the $\mathrm{CP}$ could follow up with the women later during gestation to inquire if any additional concerns with their medications have arisen.

\section{Limitations}

While the semistructure telephone interview allowed for open-ended and probing questions in order to gain a deeper understanding of participants' values and perceptions, there are limitations to our findings. Findings may be different in other study settings because of our integrated care model and inclusion of non-English-speaking women.

The CP service may not be implementable in nonintegrated outpatient practices, obstetric offices, or in a community pharmacy. While the SafeStart feasibility study in Norway found that it was feasible to provide community pharmacist consultations in early pregnancy, ${ }^{22}$ our results may not be generalizable to community pharmacists in the United States.

Biases including participation, recall, and interviewer may have been present. We attempted to limit recall and interviewer bias by contacting pregnant women within 7 days after $\mathrm{CP}$ outreach, creating an interview guide, and practicing the interviewing to decrease ambiguity. We interviewed a range of women and reached a saturation of responses in an attempt to limit participation bias. Information on preeclampsia, previous caesarean section, and prenatal vitamin and supplement use were not collected.

\section{Conclusions}

Overall, a CP medication review and education service was perceived positively by pregnant women. Three main themes emerged in the analysis: satisfaction with service, comfort with medication use during pregnancy, and connectedness to the health care team. Participants reported satisfaction in the quality, timeliness, and convenience of the service. Participants reported that it was beneficial to have their medications reviewed early in pregnancy to assist in medication use decisions before their first obstetric visits. In addition, participants described how the service led to a greater appreciation of their health care team, including the pharmacist. Future research should be conducted to assess the clinical outcomes (e.g., medication adherence, birth defects, pain management, and postpartum depression) associated with a CP medication review and education service.

\section{Authors}

MINNA LEE, PharmD, Case Management Department, Sutter Health Palo Alto Medical Foundation, Sunnyvale, California. DEANNA KURZ, BA, and KARA RIVERA, PharmD, Pharmacy Department, Kaiser Permanente Colorado, Aurora. SARAH SCHWIESOW, PharmD, Pharmacy Department, Children's Hospital Colorado, Aurora. THOMAS DELATE, PhD, MS, Drug Use Management, Kaiser Permanente National Pharmacy, Aurora, Colorado, and Clinical Pharmacy Department, Skaggs School of Pharmacy and Pharmaceutical Sciences, University of Colorado Anschutz Medical Campus, Aurora. STEPHANIE CAMPBELL, PharmD, Pharmacy Department, Kaiser Permanente Colorado, Aurora, and Clinical Pharmacy Department, Skaggs School of Pharmacy and Pharmaceutical Sciences, University of Colorado Anschutz Medical Campus, Aurora. KARI OLSON, PharmD, Pharmacy Department, Kaiser Permanente Colorado, Aurora; Drug Use Management, Kaiser Permanente National Pharmacy, Aurora; and Clinical Pharmacy Department, Skaggs School of Pharmacy and Pharmaceutical Sciences, University of Colorado Anschutz Medical Campus, Aurora.

AUTHOR CORRESPONDENCE: Thomas Delate, PhD, MS, Kaiser Permanente Colorado, 16601 E. Centretech Pkwy., Aurora, CO 80011. Tel.: 303.739.3538; Email: tom.delate@kp.org.

\section{DISCLOSURES}

This study was funded by Kaiser Permanente. The authors have nothing to disclose.

Preliminary results were presented at the Mountain States Conference for Residents and Preceptors, May 2019, in Salt Lake City, UT

\section{REFERENCES}

1. Mosley JF 2nd, Smith LL, Dezan MD. An overview of upcoming changes in pregnancy and lactation labeling information. Pharm Pract (Granada). 2015;13(2):605

2. Lupattelli A, Spigset O, Twigg MJ, et al. Medication use in pregnancy: a cross-sectional, multinational web-based study. BMJ Open. 2014;4(2): e004365.

3. Mitchell AA, Gilboa SM, Werler MM, Kelley KE, Louik C, HernándezDíaz S; National Birth Defects Prevention Study. Medication use during pregnancy, with particular focus on prescription drugs: 1976-2008. Am J Obstet Gynecol. 2011;205(1):51.el-8.

4. Haas DM, Marsh DJ, Dang DT, et al. Prescription and other medication use in pregnancy. Obstet Gynecol. 2018;131(5):789-98. 
5. Leung HY, Saini B, Ritchie HE. Medications and pregnancy: the role of community pharmacists - a descriptive study. PloS One. 2018;13(5):e0195101.

6. Lynch MM, Amoozegar JB, McClure EM, et al. Improving safe use of medications during pregnancy: the roles of patients, physicians, and pharmacists. Qual Health Res. 2017;27(13):2071-80.

7. Ragland D, Briggs GG, Wasik M, et al. Obstetrical opportunities: will pharmacy ever realize them? Ann Pharmacother. 2012;46(2):297-300.

8. Morgan MA, Cragan JD, Goldenberg RL, Rasmussen SA, Schulkin J. Obstetrician-gynaecologist knowledge of and access to information about the risks of medication use during pregnancy. J Matern Fetal Neonatal Med. 2010;23(10):1143-50

9. Ceulemans M, Lupattelli A, Nordeng H, Odalovic M, Twigg M, Foulon V. Women's beliefs about medicines and adherence to pharmacotherapy in pregnancy: opportunities for community pharmacists. Curr Pharm Des. 2019;25(5):469-82

10. Nordeng H, Ystrøm E, Einarson A. Perception of risk regarding the use of medications and other exposures during pregnancy. Eur J Clin Pharmacol. 2010;66(2):207-14

11. Samuel N, Einarson A. Medication management during pregnancy: role of the pharmacist. Int J Clin Pharm. 2011;33(6):882-85.

12. Frear RS. Patients' understanding about drugs. JAMA. 1987;257(14): $1899-900$.

13. DiPietro Mager NA. Preventing infant mortality: pharmacists call to action. J Am Pharm Assoc. 2016;56(1):82-87.

14. Ulin PR, Robinson ET, Tolley EE. Qualitative Methods in Public Health: A Field Guide for Applied Research. San Francisco, CA: Jossey-Bass; 2005

15. Ryan F, Coughlan M, Cronin P. Interviewing in qualitative research: the one-to-one interview. Int J Ther Rehabil. 2009;16(6):309-14.

16. Turner DW. Qualitative interview design: a practical guide for novice investigators. The Qualitative Report. 2010;15(3);754-60. Available at: http:// www.nova.edu/ssss/QR/QR15-3/qid.pdf. Accessed September 13, 2020.

17. Cocohoba J, Comfort M, Kianfar H, Johnson MO. A qualitative study examining HIV antiretroviral adherence counseling and support in community pharmacies. J Manag Care Pharm. 2013;19(6):454-60. Available at: jmcp.org/doi/10.18553/jmcp.2013.19.6.454.
18. Clark DO, Von Korff M, Saunders K, Baluch WM, Simon GE. A chronic disease score with empirically derived weights. Med Care. 1995;33(8):783-95.

19. Quan H, Sundararajan V, Halfon P, et al. Coding algorithms for defining comorbidities in ICD-9-CM and ICD-10 administrative data. Med Care. 2005;43(11):1130-39.

20. Glaser BG, Strauss AL. The Discovery of Grounded Theory: Strategies for Qualitative Research. Chicago, IL: Aldine; 1967.

21. Curry LA, Nembhard IM, Bradley EH. Qualitative and mixed methods provide unique contributions to outcomes research. Circulation. 2009;119(10):1442-52.

22. Truong MB, Ngo E, Ariansen H, Tsuyuki RT, Nordeng H. Community pharmacist counseling in early pregnancy-results from the SafeStart feasibility study. PLoS One. 2019;14(7):e0219424

23. Bradley CP. The future role of pharmacists in primary care. Br J Gen Pract. 2009;59(569):891-92.

24. Ragland D, Payakachat N, Hays EB, Banken J, Dajani NK, Ott RE. Depression and diabetes: establishing the pharmacist's role in detecting comorbidity in pregnant women. J Am Pharm Assoc. 2010;50(2):195-99.

25. Evans E, Patry R. Management of gestational diabetes mellitus and pharmacists' role in patient education. Am J Health Syst Pharm. 2004:61(14):1460-65.

26. Santucci AK, Gold MA, Akers AY, Borrero S, Schwarz EB. Women's perspectives on counseling about risks for medication-induced birth defects. Birth Defects Res A Clin Mol Teratol. 2010;88(1):64-69.

27. Hammer RP, Burton-Jeangros C. Tensions around risks in pregnancy: a typology of women's experiences of surveillance medicine. Soc Sci Med. 2013:93:55-63.

28. Reid B, Sinclair M, Barr O, Dobbs F, Crealey G. A meta-synthesis of pregnant women's decision-making processes with regard to antenatal screening for down syndrome. Soc Sci Med. 2009;69(11):1561-73.

29. Jasper JD, Goel R, Einarson A, Gallo M, Koren G. Effects of framing on teratogenic risk perception in pregnant women. Lancet. 2001;358(9289):1237-38 